\title{
Modeling and Analysis of Linkage Fluctuation Network for Complex Industrial Process
}

\author{
Jian-min Gao \\ State Key Lab for Manufacturing Systems Engineering, \\ Xi’an Jiaotong University, Xi'an 710049, China \\ gjm@mail.xjtu.edu.cn
}

\author{
Zhi-yong Gao \\ State Key Lab for Manufacturing Systems Engineering, \\ Xi'an Jiaotong University, Xi'an 710049, China \\ zhygao@mail.xjtu.edu.cn
}

\author{
Jun-tai Xie \\ State Key Lab for Manufacturing Systems Engineering, \\ Xi'an Jiaotong University, Xi'an 710049, China \\ xiejuntai@126.com \\ $\mathrm{Xu}$ Gao \\ State Key Lab for Manufacturing Systems Engineering, \\ Xi'an Jiaotong University, Xi'an 710049, China \\ gaoxu@tpri.com.cn
}

\begin{abstract}
Industry process includes a variety of monitoring variables, there are strong relevance between variables. Time series from different monitoring points can reflect the relationship of system's linkage fluctuation. Using coarse graining method, monitoring series has been changed into a sequence of characters. The sequence which is sliding by a certain length has been converted into a continuous modals of linkage fluctuation. The complex network of linkage fluctuation is composed of all the modals and the link edges between them. Transformation law for modals has been analyzed by degree distribution and structure entropy of complex networks theory. This method has been applied to analyze typical fault of monitoring data from large-scale coal chemical compressor units. It is indicated that the modals both in the normal operation and fault condition follows a powerlaw distribution, transmission is mainly finished by few modals, but the structure entropy of network have obvious difference. The results not only can be used for complex industrial process fault warning but also provides an idea for assessment of process recovery.
\end{abstract}

Keywords-Industry process; Linkage fluctuation; Modeling and analysis; Complex network; Structure entropy

\section{INTRODUCTION}

Modern process industry system is a distributed and complex electromechanical system, which has numerous sets of electromechanical equipment [1-3], the coupling degree of system internal relevancy is high, equipment failure and process adjustment easily lead to systemic fluctuations. It is important to find the fault in the process operation timely and accurately, particularly in reasonably regulating and controlling the system on the upstream and downstream. Dispatchers often depend on operation experience in scheduling the upstream and downstream, for the sake of safety over scheduling and not timely scheduling can easily lead the interruption of production or the decline of production load, which may cause huge economic losses. Analysis of correlation fluctuation for time series from the distributed control system is an ideal method. If the information of correlation fluctuation between monitoring variables in process is timely obtained, the fast and precise scheduling for the system will be realized and the safety level of operation for production process will be improved.

Time series analysis has been widely used in many fields, such as financial, economic, meteorology and engineering management, effective information that is fully excavated from time series will be useful to managers for management and decision-making [4-11]. Time series analysis includes univariate analysis and multivariate analysis, analysis of industry process which contains many variables belongs to the latter.

Industrial process is a complex and nonlinear dynamic system, the changes of process monitoring variable caused the changes of correlation fluctuation among variables, the deterioration of correlation fluctuation usually reflect the fault of the process. Traditional method in research on the relationship among the variables which mainly focuses on exploring the equilibrium relationship between them is difficult to reveal the correlation fluctuation mechanism among variables [5-7]. Learning from physical economics theory, the coarse grain method combined with complex network theory has been achieved beneficial effect in analysis of correlation fluctuations for time series [8-11].

The study of the correlation fluctuation of multivariable is based on two variables. In this paper, the complex network theory and the coarse-grained method has been applied to research correlation fluctuations in process industry. Using coarse-grained method, the correlation fluctuation relationship of time series from the two monitoring points on the upstream and downstream of fault point has been converted into correlation fluctuation modal, order relations for edge, a complex network model of correlation fluctuation has been constructed. Using the complex network theory, the fluctuation correlation network has been analyzed, the law of inherent variation in system has been studied, which can provide references for the fault's early warning and the assessment of process recovery. 


\section{The CONSTRUCtion METHOD FOR CORRELATION FLUCTUATION NETWORK}

\section{A. Symbolic of Correlation Fluctuation for Monitoring Series}

The symbolization of time series' fluctuation correlation is the effective way of abstraction for correlation relationship [10], it is critical for the analysis of correlation fluctuation for time series to set reasonable modal symbols.

Assuming that $A_{t}$ is the sampling value of the time series $A$ at sampling period $t$ and the meaning of $A_{t-1}$ is the sampling value of the time series $A$ at sampling period $t-1$. $\Delta A$ is the change of the sampling value at sampling period $t$ and $t-1$. The meaning of $B_{t}, B_{t-1}$ and $\Delta B$ are same as the above. The expression of $\Delta A$ and $\Delta B$ is listed in formula (1).

$$
\left\{\begin{array}{l}
\Delta A=A_{t}-A_{t-1} \\
\Delta B=B_{t}-B_{t-1}
\end{array}\right.
$$

The symbolic series $T C_{i}$ of correlation fluctuation for monitoring series as shown in formula (2).

$$
T C_{\mathrm{i}}= \begin{cases}P & \text { If } \Delta \mathrm{A} \times \Delta \mathrm{B}>0 ; \\ N & \text { If } \Delta \mathrm{A} \times \Delta \mathrm{B}<0 ; \\ I & \text { If } \Delta \mathrm{A} \times \Delta \mathrm{B}=0\end{cases}
$$

In formula (2), the symbol $P$ means that time series A and $\mathrm{B}$ is in positive correlation fluctuation; the symbol $N$ means that time series $\mathrm{A}$ and $\mathrm{B}$ is in negative correlation fluctuation between time series A and B; the symbol $I$ means that time series $A$ and $B$ is in independent fluctuation.

Using the above method of symbolic, correlation fluctuation between the variables can be represented with a continuous sequence of symbols, so the study of the coarsegrained symbol sequence is equivalent to original series' research. Symbol sequence $T C$ after transformed is shown in formula (3).

$$
T C=\left(T C_{1}, T C_{2}, \cdots, T C_{\mathrm{n}}\right), T C \in(P, I, N)
$$

\section{B. Construction Method of Network for Correlation Fluctuation}

After symbolizing the correlation fluctuation of the time series, an abstract symbol sequence is formed. Taking a certain length symbols sequence as a modal and sliding data with a certain length as one step, the continuous modal of correlation fluctuation is formed. Due to the modal formation process is realized in the form of a sliding data, a modal form is based on a modal, modal between transitivity is beneficial to construct complex network.

Complex network model of correlation fluctuation for time series is a directed and weighted networks, whose node is the modal of correlation fluctuation and edge is the directed transformation between the modals, times of transition between the modals is the weight of the directed and weighted network.

\section{ANALysis METHOD OF CORRELATION FLUCTUATION NETWORK}

The analysis of complex network for the correlation fluctuation between the variable is used to explain the relationship of the various modals' distribution characteristics and their mutual conversion relationship. There are a number of important statistical parameters in complex network theory, such as degree, intensity, the cumulative degree distribution, network structure entropy. Through the analysis of these parameters, the relationship of fluctuation correlation and the change between variables rule have been mastered, those can reasonably predict the trends for the state of related fluctuations in the future.

\section{A. Statistical Method of Correlation Fluctuation Modal}

The complex network of correlation fluctuation for process is a directed and weighted networks, the edge of the network contains the information of weight. The intensity and intensity distribution can describe the correlation fluctuation between the modals.

The calculation method of point intensity $[10,12]$ is listed in formula (4).

$$
k_{i}=\sum_{j=1}^{N_{i}} W_{i j}
$$

In formula (4), $N_{i}$ is a collection of all the neighboring nodes which connect to node $i$ and point from the node $i, W_{i j}$ is the weight of the edge that node $i$ point to node $j$. Strength is greater, it is indicated that the status of modal in complex network is more important than others and the modal converts to other modals more times.

The intensity distribution of different node is different due to different intensity. The calculation method of The intensity distribution is shown in formula (5).

$$
P\left(k_{i}\right)=n k_{i} / \sum_{i=1}^{N} k_{i}
$$

In formula (5), $n$ is the number of the node whose intensity equals $k_{i}, \mathrm{~N}$ is the total number of all the nodes. The greater of the node's intensity distribution means the more transformation of the current modal to the other modal, the greater probability of the node appears in the network, the modal is more important than others.

\section{B. The Change Rules of Correlation Fluctuation Modal}

The complex relationship of the correlation fluctuation modals can be described by the statistical rules of the related fluctuation modals, and it can be expressed as the change rule of correlation fluctuation modals in the time course. 
The actual complex network have a high connectivity degree of the central node and a large number of low connectivity degree of node, this is the no scale (or powerlaw) property of network. Entropy is a disordered metric for system, structure entropy of complex network based on the degree distribution can measure the complex network ordered state succinctly. The definition [13] of network's structure entropy is listed as the formula (6).

$$
E=\sum_{i=1}^{N} P\left(k_{i}\right) \cdot \ln P\left(k_{i}\right)
$$

Standard structure entropy [13] of complex network has been adopted to eliminate the influence of the nodes' number $\mathrm{N}$ on the entropy $\mathrm{E}$, the expression of standard entropy for the complex network is listed as the formula (7).

$$
\hat{E}=\frac{-2 \sum_{i=1}^{N} P\left(k_{i}\right) \cdot \ln P\left(k_{i}\right)-\ln 4(N-1)}{2 \ln N-\ln 4(N-1)}
$$

\section{MOdELING AND ANALYSIS OF THE CORRELATION FLUCTUATION NETWORK FOR INDUSTRIAL PROCESS}

Compressor unit as the typical unit to process industries is one of the most important part which operates in a safety state for the whole process of industrial production process and stable operation. When the raw material is stable, the material status in producing process can indirectly reflect the industrial process running status, the information of industrial process fault can be obtained from the fluctuations of material status, so the typical process fault data can be applied to the evaluation of process fault warning and process restoration.

\section{A. Monitoring Time Series and Its Pretreatment Methods}

In this paper, a coal chemical enterprise compressor unit parking in front of DCS system in process monitoring data, the process of the accident described as: due to the compression of air compressor turbine shaft seal leakage, high temperature gas radiation to the electro-hydraulic converter, electro-hydraulic converter signal cable is baked caused by fault signal, eventually leading to high valve to the no action, the crew was forced to hand lever parking.

Selection two variables (such as variable names PI7603: air compressor exhaust pressure and PI7611: booster 1 section of the inlet pressure) that are closely related to the failure of process as the basis data of analysis, the feasibility of the method has been verified through the analysis.

The time series data of actual monitoring process from the distributed control system often contains a lot of noise that affect the analysis results, so the original series data need noise reduction. High frequency noise can be significantly observed in the series as shown in Fig. 1a. Different variables take proper wavelet function and decomposition layers. Using fixed threshold method, the detail coefficients of wavelet are decomposed with the soft threshold, and then the approximate coefficients of last layer and all detail coefficient are reconstructed, the curve after noise reduction has been shown in Fig. 1b. Comparing Figure 1a and Fig. 1b, the curve after noise reduction keeps the change trend of the original data and eliminate noise in high frequency, the curves after the processing of noise reduction are more smooth than the curve formed with original data. The data has been used standardization method in order to facilitate comparison.

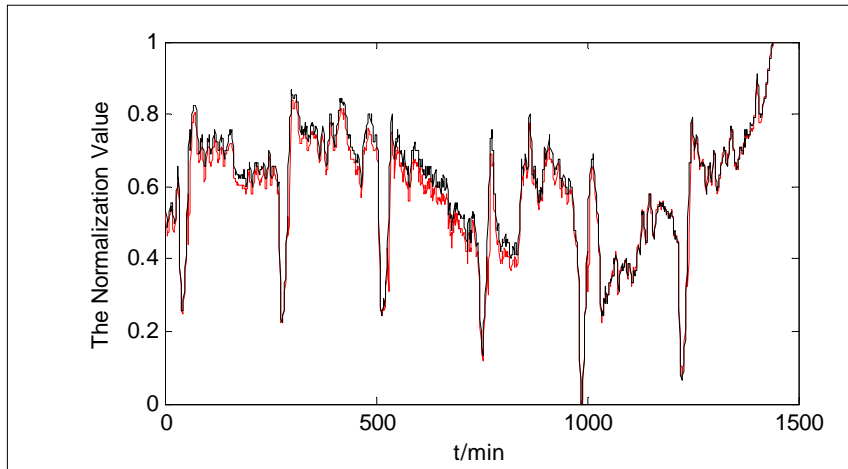

(a) The original time series

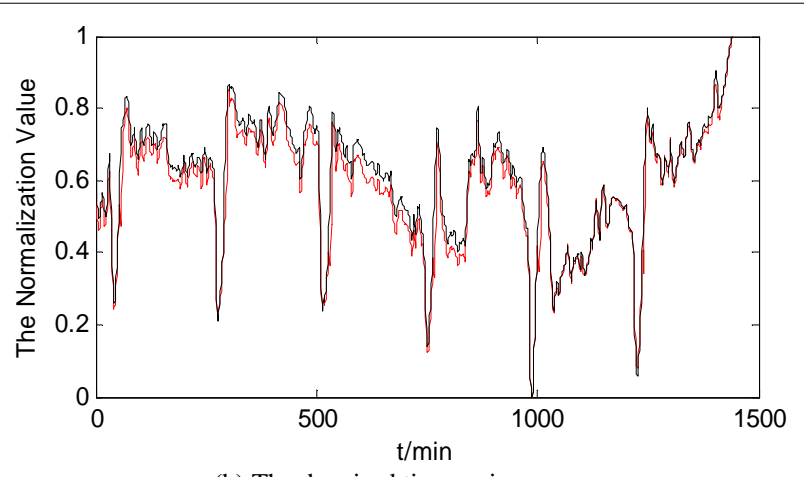

(b) The denoised time series

Figure 1. The curve of monitoring variables PI7602 and PI7611

\section{B. Granularity of Selection and Treatment Process for the Series of Correlation Fluctuation}

If the correlation fluctuation of two series is represented by the symbols, the same number of abstract symbol sequence can be obtained. Taking five continuous symbols of correlation fluctuation as a modal and selecting the length of one sampling as the size of step for data sliding, a series of correlation fluctuation modal for two time series can be obtained. Taking data in May 10, 2010 for an example, the data slide and modal forming process has been shown in Table I. 
TABLE I. The CoArse Graining Process of CORRElation FluCtuAtion For SERIES A AND B

\begin{tabular}{|c|c|c|c|c|c|c|}
\hline \multirow{2}{*}{ No. } & \multicolumn{2}{|c|}{ Series A } & \multicolumn{2}{|c|}{ Series A } & \multicolumn{2}{|c|}{ Symbols and Modals of correlation fluctuation } \\
\hline & Series value & variation & Series value & variation & Symbolic & Modal \\
\hline 1 & 0.5150 & & 0.0934 & & & \\
\hline 2 & 0.5235 & 0.0085 & 0.1307 & 0.0373 & $\mathrm{P}$ & \\
\hline 3 & 0.5414 & 0.0179 & 0.1024 & -0.0283 & $\mathrm{~N}$ & \\
\hline 4 & 0.5048 & -0.0366 & 0.0973 & -0.0051 & $\mathrm{P}$ & \\
\hline 5 & 0.4953 & -0.0095 & 0.1795 & 0.0822 & $\mathrm{~N}$ & \\
\hline 6 & 0.5631 & 0.0678 & 0.0988 & -0.0807 & $\mathrm{~N}$ & PNPNN \\
\hline 7 & 0.4732 & -0.0899 & 0.1465 & 0.0477 & $\mathrm{~N}$ & NPNNN \\
\hline 8 & 0.4715 & -0.0017 & 0.1387 & -0.0078 & $\mathrm{P}$ & PNNNP \\
\hline 9 & 0.4287 & -0.0428 & 0.2104 & 0.0717 & $\mathrm{~N}$ & NNNPN \\
\hline 10 & 0.4478 & 0.0191 & 0.1196 & -0.0908 & $\mathrm{~N}$ & NNPNN \\
\hline$\ldots$ & $\ldots$ & $\ldots$ & $\ldots$ & $\ldots$ & $\ldots$ & $\ldots$ \\
\hline 1440 & 0.482 & -0.0667 & 0.7065 & 0.1087 & $\mathrm{~N}$ & NNPNN \\
\hline
\end{tabular}

\section{The Statistical Regularity of Correlation Fluctuation} Modal

The use of the above sequence fluctuation correlation of coarse grain method fault appeared the six day's data before processed, and statistics of the sequence and modal of correlation fluctuation is shown in Table II.

TABLE II. STATISTICS FOR THE SYMBols AND THE MODALS OF CORRELATION FLUCTUATION

\begin{tabular}{|c|c|c|c|c|c|c|c|}
\hline \multirow{2}{*}{ Date } & \multicolumn{3}{|c|}{ The Number of Symbols } & \multicolumn{3}{|c|}{ The Percentage of the Symbols } & \multirow{2}{*}{$\begin{array}{c}\text { Modal } \\
\text { The number of modal }\end{array}$} \\
\hline & $n(P)$ & $n(N)$ & $n(I)$ & P\% & $N \%$ & $I \%$ & \\
\hline 2010-05-07 & 1323 & 107 & 9 & 91.94 & 7.436 & 0.6254 & 55 \\
\hline 2010-05-08 & 1295 & 136 & 8 & 90.00 & 9.451 & 0.5560 & 42 \\
\hline 2010-05-09 & 973 & 466 & 0 & 67.62 & 32.38 & 0 & 32 \\
\hline 2010-05-10 & 698 & 689 & 52 & 48.51 & 47.88 & 3.614 & 125 \\
\hline 2010-05-11 & 745 & 645 & 49 & 51.78 & 44.82 & 3.405 & 115 \\
\hline 2010-05-12 & 663 & 720 & 56 & 46.07 & 50.03 & 3.892 & 126 \\
\hline
\end{tabular}

Analysis of the six day's data before the accident shows that the percentage of positive fluctuation correlation symbol $P$ from the total sequence show clear downward trend as shown in Table II, the negative correlation fluctuation symbol $N$ accounted for the percentage of the total length of the sequence presents a significant trend, and no correlation fluctuation symbol $I$ accounted for a lower percent of the total length of the sequence of the it indicated that the relevance of two sequences is strong in line with the actual situation. Fluctuation correlation statistical modal overall from the point of view, theory by three five symbols fluctuation correlation modal has $3^{5}=243$ species, but actual are significantly less, which show that random fluctuation of the two variables under certain coupling constraints, the total of correlation fluctuation modal presents a growth trend.

\section{Study on the Changes of Correlation Fluctuation Modal}

The curves of relationship for point intensity $K$ and the accumulation of intensity distribution $L P(k)$ follow a powerlaw distribution as shown in Fig. 2 in the correlation 
fluctuation network which has been formed with the six days' data from compressor unit.
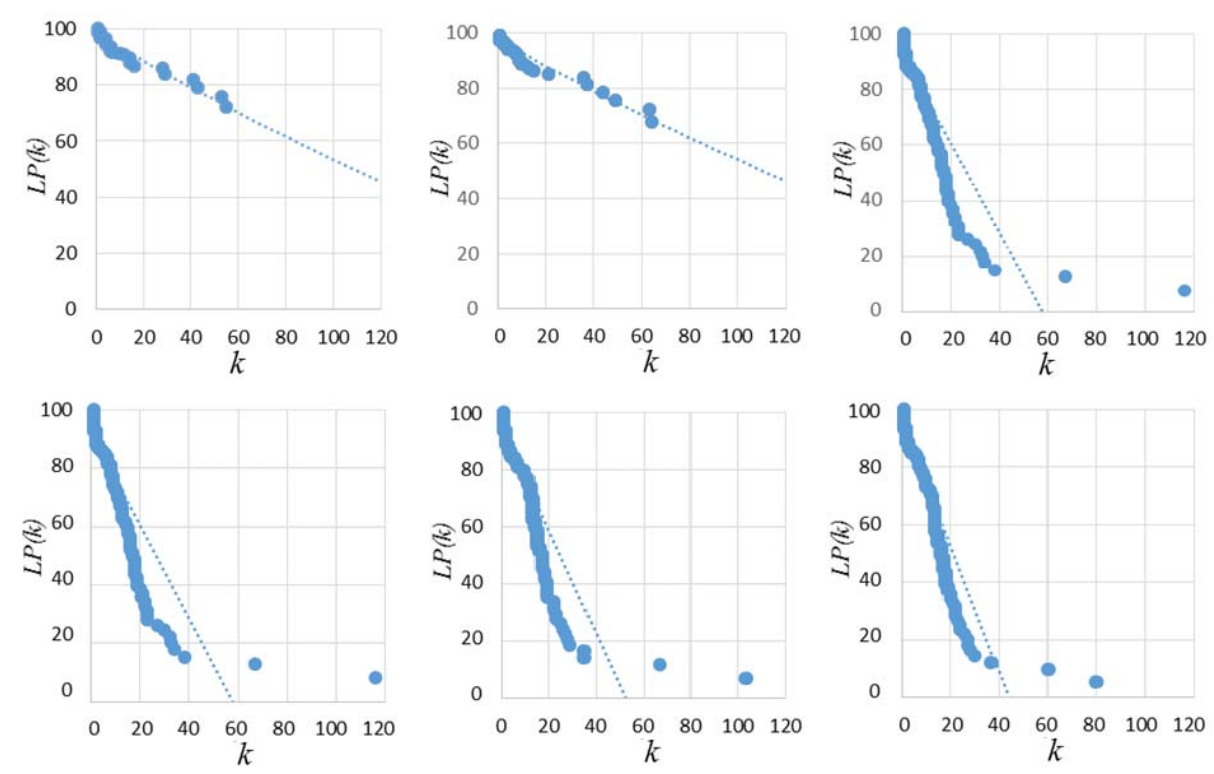

Figure 2. The curve of relationship for point intensity and cumulative intensity distribution

The point intensity's distribution of correlation fluctuation can be seen the growth of decline as shown in Fig. 2, the trend line of the curve which decreased significantly show continued deterioration of the linkage of the two nodes, it indicates that the probability of the compression unit's failure increase.

The network of correlation fluctuation between variable PI7602 and variable PI7611 has been shown in Fig. 3 when the compression generator was in the normal work.

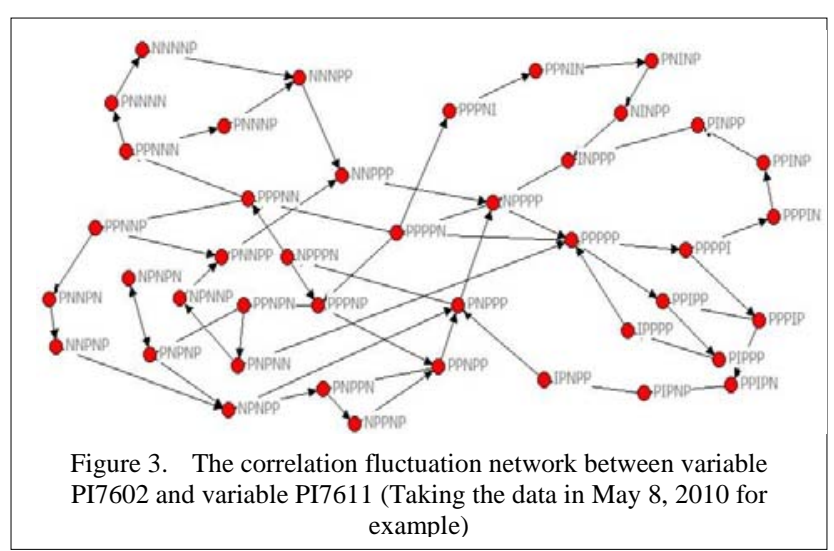

From Fig.3 few modal (such as: $p p p p p, N P P P P, P P P N P$ and $P N P P P$ ) have more connections and the rest of the modal contains only a few connections, the power-law characteristics of network has been intuitively descripted, the result is consistent with the above analysis.

In order to quantitative the change of the correlation fluctuation's state, the structure entropy of complex network has been introduced to quantitatively describe the comovement of decline. According to the formula (6), the structure entropy of correlation fluctuation network has been calculated and the trend of the structure entropy has been shown in Fig. 4 based on the six day's data before the accident.

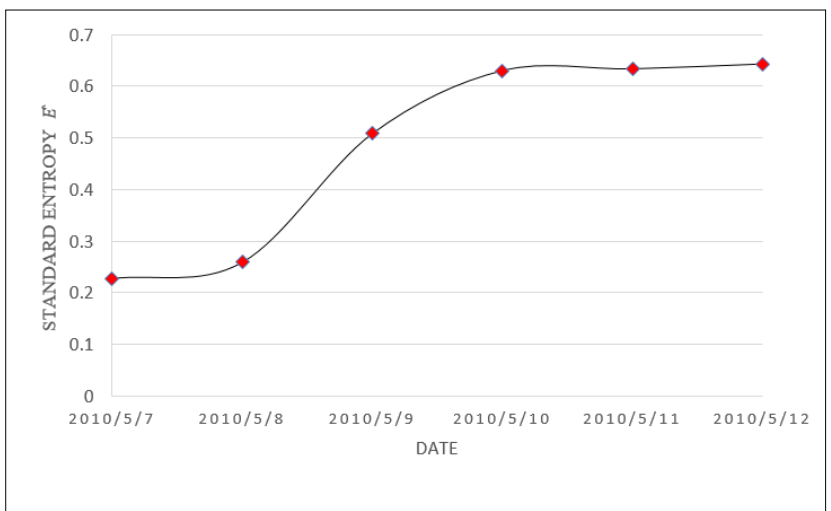

Figure 4. The variation trend of the network structure entropy of the

Analyzing the six day's data before the accident, the structure entropy of correlation fluctuation network between the variables presents a continuous growth trend as shown in Fig. 4, which indicates that the risk of the failure associated with this variable in the compressor unit increases. So in order to maintain the normal operation of the system, the structure entropy of the correlation fluctuation network between variables must be maintained in a certain range, the system can maintain the stable working condition. From the type of the accident, the fault that belongs to the ramp fault is in line with the actual situation. 
The structure entropy of correlation fluctuation network between variables as shown in Fig. 4 has been further analyzed. It is found that the value of structure entropy for correlation fluctuation network between variables is small at first (Such as May 7-8, 2010), it means that the transformation of the correlation fluctuation modals remains relatively stable, the compressor units is in a good working condition. The structure entropy of correlation fluctuation network between variables gets higher (Such as May 7-12, 2010), it means that the uncertainty of the conversion for correlation fluctuation modals increases, it is indicated that the compressor unit running in a poor state and the possibility of random accidents increases. While in the middle of the transition state, the compressor units maintain the original state will be changed (Such as May 9, 2010). The change can be regarded as a sign of the abnormal transformation for correlation fluctuation modals in a certain degree. It is of great significance to grasp the transition state, it mainly reflects in favor of grasping the law of the state change for monitoring points. This will win more time to take measures for the abnormal situation in the operation of the system and avoid the continuous development of the fault which will cause greater damage.

\section{FOUNDATION ITEM(S)}

Project supported by the National Science and Technology Support Plan of China under Grant (No.2012BAF12B04)

*Communication author: Zhi-Yong Gao E-mail: zhygao@mail.xjtu.edu.cn

\section{ACKNOWLEDGMENT}

Modeling and analysis of correlation fluctuation network for complex industry process, the method of the complex networks combined with coarse graining method is applied to the correlation fluctuation research in the industry process.

(1) The method of coarse graining has been adopted to translate the relationship of the correlation fluctuation between monitoring time series from complex industrial process into the correlation fluctuation modals. The complex network model of associated volatility process has been constructed with the modals as the nodes and the relationship of the modals' transformation for edge. This method can easily characterize the relationship of the correlation fluctuation between variables.

(2) Using the degree distribution theory of complex network to analyze the correlation fluctuation modals between variables in the industrial process, it can be found that the correlation fluctuation network follows power-law distribution in the industrial process.

(3) A new method has been proposed that network's structure entropy has been adopted to study the inherent variation of the correlation fluctuation between variables in industry process, the change of the correlation between the variables has been quantitatively expressed that providing decision support for the fault's warning and the assessment of process recovery.

Of course, this paper focuses on the verification of the method introduced in the study, the coarser granularity has been selected. In order to obtain the better study effect, finer granularity can be used to describe the correlation fluctuation relationship in the research of the later stage. This paper only studies the relationship of correlation fluctuation for the variables which are closely related to the fault, the correlation fluctuation of the whole industrial process need description in the practical application, there are some further research on development and application for the method in order to study the running state of the system more comprehensively.

\section{REFERENCES}

1] Huang XL, Gao JM, Jiang HQ, Chen K. A Systematic Fault Root Causes Tracing Method for Process Systems [C]. Porceeding of the Annual Reliability and Maintainability Symposium, 2011, 24-27: 1-7.

[2] SUN Kai, GAO Jianmin GAO Zhiyong. Health state analysis of modern industry system through system color picture based on the data-driven [J]. Chinese Journal of Mechanical Engineering, 2012, 18(48): 186-191.

[3] Gao Zhi yong, Huo Wei han, Gao Jian min, Jiang Hong quan. [J]. Diffusion mapping and abnormal recognition algorithm for mass data of chemical system [J]. Computer Integrated Manufacturing Systems, 2014, 12(20): 3092-3096.

[4] Pandit S M, Wu S M. Time series and system analysis with application [M]. New York: Wiley, 1983: 313-354.

[5] Yen H L, Hsu Ning H. Jump dynamics with structural breaks for crude oil prices [J]. Energy Econ, 2012, 32: 343-350.

[6] Svetlana M, Russell S Energ. Cointegration between oil spot and future prices of the same and different grades in the presence of structural change [J]. Energy Policy, 2009, 37: 1687-1693.

[7] Kaufmann R K, Ullman B. Oil prices, speculation, and fundamentals: Interpreting causal relations among spot and future prices [J]. Energy Economics, 2009, 31(4): 550-558.

[8] Zhou Lei, Gong Zhi qiang, Zhi Rong, Feng Guo lin. An approach to research the topology of Chinese temperature sequence based on complex network [J]. Chinese Journal of Physics, 2008, 57(11): 7380-7389.

[9] Chen Wei dong, Xu Hua, Guo Qi. Dynamic analysis on the topological properties of the complex network of international oil prices. Chinese Journal of Physics, 2010, 7(59): 4514-4523.

[10] An Hai gang. Linkage Fluctuation in Double Variables of Time Series Based on Complex Networks [J]. Chinese Journal of Computational Physics, 2014, 6(31): 742-750.

[11] Zhou L, Gong Z Q, Zhi R, Feng G L. Influence of time delay on global temperature correlation [J]. Acta Phys Sin, 2011, 20(8): 380387.

[12] Yook S H, Jeong H, Barabási A L, Tu Y. Small world network of athletes: Graph representation of the world professional tennisplayer [J]. Phys Rev Lett, 2010, 86: 5835-5838.

[13] Tan Yue jin, Wu Jun. Network structure entropy and its application to scale-free networks [J]. Systems Engineering-Theory \& Practice, 2004, 24(6): 1-3. 